\title{
Analysis of stripe design in the clothing based on eye tracking
}

\author{
Shurong $\mathrm{Hu}^{1, a}$, Bei Xiao ${ }^{2, b}$ \\ ${ }^{1}$ Fashion Institute, Zhejiang University of Science and Technology, Hangzhou, 310023, China \\ ${ }^{2}$ Fashion College, Zhejiang Sci-tech University, Hangzhou, 310008, China \\ arongerdavy@126.com, bzjxu_liu@126.com
}

Keywords: Stripe design; disabled clothing; eye tracking.

Abstract. In order to reduce the impact on the psychology of appearance defects for upper limb disabled, this paper tried to weaken the physical defect characteristics, using stripe design to transfer focus on defective parts of the disabled. The parameters of horizontal and radiation stripes were confirmed and the clothing was designed. Based on the eye tracking experiment, the effect of stripe parameters on visual transfer was discussed. The result can be a guideline for disabled clothing design.

\section{Introduction}

In addition to meet the special physiological needs of the function, appearance and styles of the clothing for disabled also need to weaken their physical defect characteristics, to reduce the impact on the psychology of appearance defects, improving self-confidence. There are some companies in abroad sell specialized clothing for the disabled on ecommerce ${ }^{[1]}$. Researchers mostly focus on the functional and comfortable clothing for disabled. Some researchers ${ }^{[2]}$ studied how to promote the convenience of wearing disabled clothing which is the same style as the normal clothing. Renee Weiss Chase ${ }^{[3]}$ proposed that the patterns using in the clothing can attract the people's distraction on the zones where the pattern used, and the imperfect parts will be not paid attention, and the accessories used also can be achieved the same purpose of diverting attention.

This paper based on the upper limb disabled clothing, and the stripe design rule to weaken the physical defects characteristic is proposed according to the eye tracking experiment. It provides a design suggestion for improving the appearance aesthetic needs of the physically handicapped.

\section{Experiment}

Clothing design and stripe parameters. This paper took the white T-shirt with short sleeve as the sample, which length was $60 \mathrm{~cm}$, bust girth was $96 \mathrm{~cm}$, waist girth was $90 \mathrm{~cm}$, shoulder width was $39 \mathrm{~cm}$ and sleeve length was $10 \mathrm{~cm}$. The T-shirt was divided into four parts A-D as shown in Fig.1. The stripe was black. Supposed the left arm was incomplete, the clothing should attract the vision to right arm (zone A).

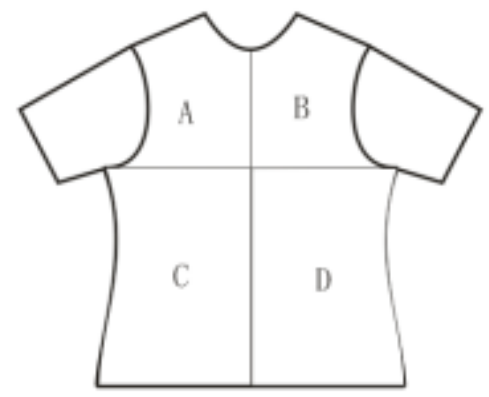

Fig. 1 T-shirts and 4 divided parts

T-shirt was designed in line with several variation parameters of the stripe. The categories of the stripe were horizontal and radiation. The horizontal stripe parameter included stripe length, density 
between stripes. The radiation stripe parameter included angle between two stripes, focus point location.

The length variation in horizontal stripe was concentrated in zone A. The length variation was defined by three levels, which were increase, decrease and stable. The length was less than the centre front line. The variation of density between stripes was defined by two levels, which were increase and decrease. The variation of angle between two stripes in radiation stripe was defined by three levels, which were increase, decrease and stable. The focus point location was defined by the intersection point of across back and waist line O1, the midpoint of armhole curve $\mathrm{O} 2$ and the shoulder point O3, respectively. The horizontal and radiation stripe styles of $\mathrm{T}$ shirt are shown in Fig.2 and Fig.3.

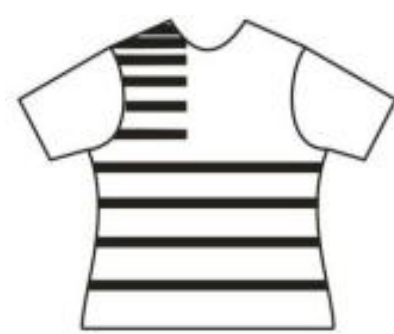

(a)

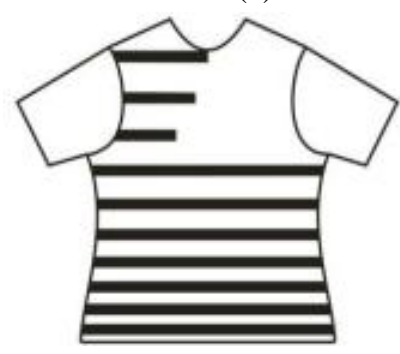

(d)

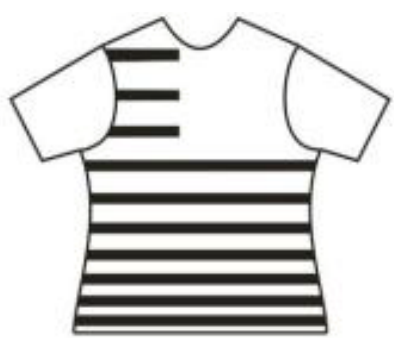

(b)

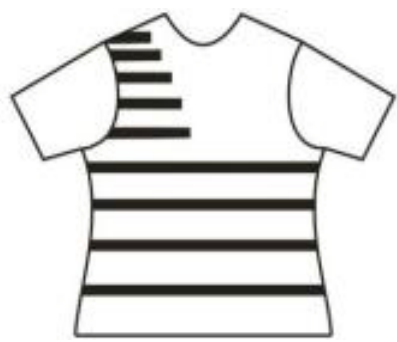

(e)

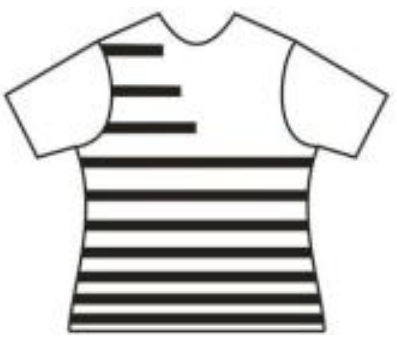

(c)

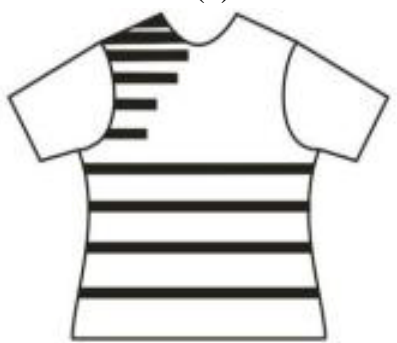

(f)

Fig.2 T-shirts with horizontal stripe

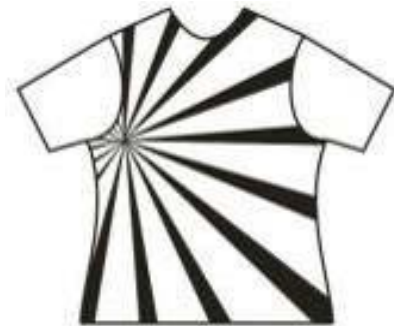

(a)

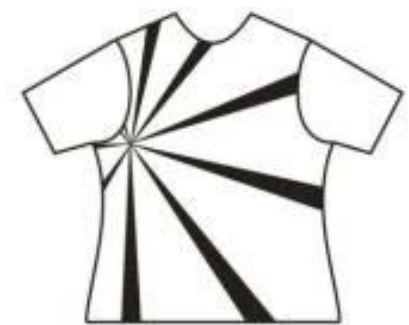

(d)

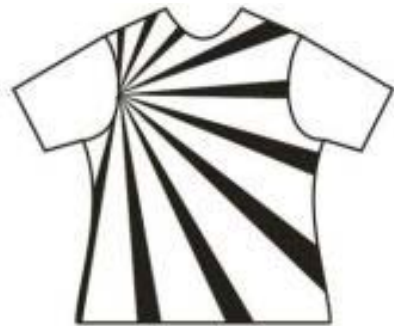

(b)

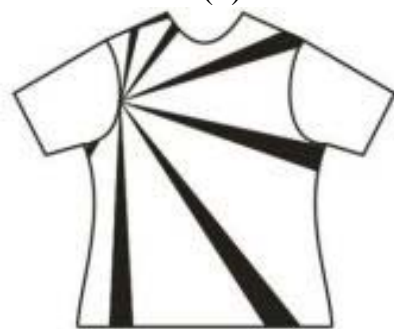

(e)

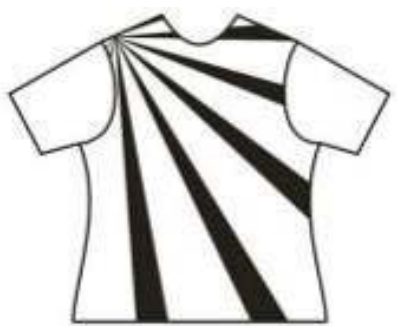

(c)

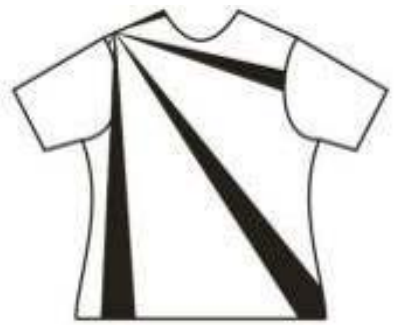

(f)

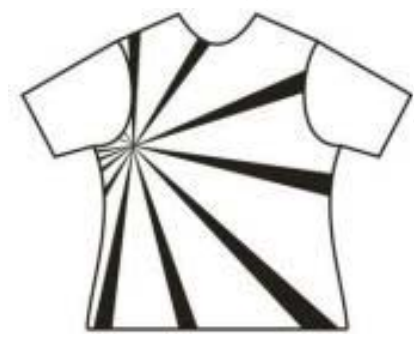

(g)

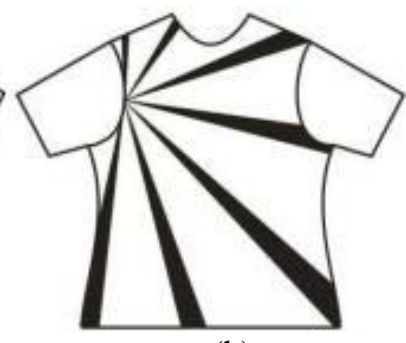

(h)

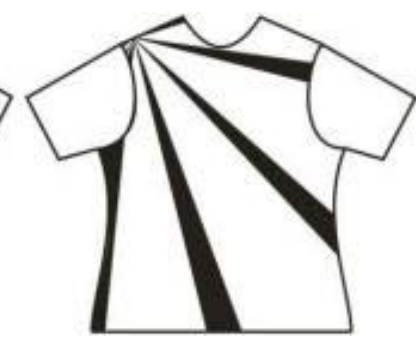

(i)

Fig.3 T-shirts with radiation stripe 
Eye tracking experiment.An eye movement experiment was conduct to measure vision concentrate area for stripe T-shirt. The equipment was Eyelink1000. 43 volunteers (21males and 22 females, $23 \pm 3$ years old ) were recruited to participate in the experiment. The total fixation duation, first fixation duration, fixation time, gaze duration and fixation point distribution were recorded. The ANVOA and least significant difference were used to analyze the experiment data.

\section{Results and Analysis}

The results of the horizontal stripe T-shirts experiment analysis were shown in Table 1 and 2.

Table 1 The horizontal strip of T-shirts analysised by ANOVA and LSD

\begin{tabular}{cccc}
\hline Parameters & Variation & sig. & LSD sig.<0.05 \\
\hline Total & Interest zone & 0.000 & A\&B, A\&C, A\&D; B\&C, B\&D \\
fixation & Density*interest zone & 0.001 & - \\
duaration & Length* interest zone & 0.023 & - \\
& Density *lenght*interest zone & 0.009 & - \\
First fixation & Interest zone & 0.000 & A\&B, A\&C, A\&D \\
duaration & Density *interest zone & 0.033 & - \\
Fixation time & Interest zone & 0.000 & A\&B, A\&C, A\&D; B\&C, B\&D \\
& Density *interest zone & 0.000 & - \\
& Length* interest zone & 0.027 & - \\
\hline
\end{tabular}

From the Table1, the difference interest zone in fixation was significant. Zone A and B were different with other zones. Total fixation duration was most different among the result data. The difference of total fixation time for density interest zone was significant. It means that the fixation time is more duration under the same stripe length.The difference of total fixation duration for length of interest zone was significant. This demonstrates the fixation time is decline with the same stripe length under the same stripe density. The difference of total fixation time for density, length, interest zone was significant. It means that the fixation duration of length variation at Zone A was increased when density was increased according to Table2.

Table 2 Total fixation duration of the horizontal strip T-shirts

\begin{tabular}{|c|c|c|c|c|c|c|}
\hline \multirow[b]{2}{*}{ Style } & \multicolumn{2}{|c|}{$\begin{array}{l}\text { Stripe design } \\
\end{array}$} & \multicolumn{4}{|c|}{ Total fixation duration } \\
\hline & $\begin{array}{c}\text { Stripe density } \\
\text { level }\end{array}$ & $\begin{array}{c}\text { Sripe lenght } \\
\text { level }\end{array}$ & ZoneA & ZoneB & ZoneC & ZoneD \\
\hline $\mathrm{a}$ & 1 & 0 & 2598.44 & 814.33 & 362.88 & 299.28 \\
\hline $\mathrm{b}$ & -1 & 0 & 1807.77 & 701.72 & 855.91 & 683.37 \\
\hline c & -1 & -1 & 2414.67 & 797.91 & 384.51 & 695.77 \\
\hline $\mathrm{d}$ & -1 & 1 & 2637.65 & 762.53 & 308.19 & 408.35 \\
\hline e & 1 & -1 & 2687.74 & 482.47 & 431.12 & 471.23 \\
\hline $\mathrm{f}$ & 1 & 1 & 2686.12 & 562.95 & 332.28 & 408.77 \\
\hline
\end{tabular}

It showed that zone A attracted more people's vision, especially when the stripe length and density were variation. The increase of stripe density can enhance the sight concentration obviously.

The effects of the radiation stripe in T-shirts are shown in Table 3 and 4.

Table 3 ANOVA and LSD analysis of the radiation strip T-shirts

\begin{tabular}{|c|c|c|c|}
\hline Parameters & Variation & sig. & LSD sig. $<0.05$ \\
\hline Total & Interest zone & 0.000 & $A \& B 、 A \& C, A \& D ; B \& D$ \\
\hline fixation & Focus point location *interest zone & 0.000 & - \\
\hline duaration & Interest zone & 0.000 & $\mathrm{~A} \& \mathrm{~B}, \quad \mathrm{~A} \& \mathrm{C}, \quad \mathrm{A} \& \mathrm{D}$ \\
\hline & Angle & 0.730 & $0 \& 1, \quad$ sig. $=0.020$ \\
\hline $\begin{array}{l}\text { First fixation } \\
\text { duaration }\end{array}$ & $\begin{array}{l}\text { Focus point location } \\
\text { Focus point location } * \text { interest zone }\end{array}$ & $\begin{array}{l}0.592 \\
0.000\end{array}$ & O1\&O2, sig. $=0.004 ;$ O1\&O3, sig. $=0.032$ \\
\hline Fixation time & Interest zone & 0.000 & $\mathrm{~A} \& \mathrm{~B}, \quad \mathrm{~A} \& \mathrm{C}, \mathrm{A} \& \mathrm{D} ; \mathrm{B} \& \mathrm{D}$ \\
\hline & Focus point location $*$ interest zone & 0.000 & - \\
\hline
\end{tabular}


As shown in Table3, the difference interest zone in fixation was significant. Zone A were different from other zones, zone B was different from zone D. The total fixation time was different from the others, this means the radiation stripe effect attracting people's sight, the vision transferred from other zone to zone A.

Table 4 Total fixation duration of the radiation strip T-shirts

\begin{tabular}{|c|c|c|c|c|c|c|}
\hline \multirow[b]{2}{*}{ Style } & \multicolumn{2}{|c|}{ Stripe design } & \multicolumn{4}{|c|}{ Total fixation duration } \\
\hline & Angle level & $\begin{array}{l}\text { Focus point } \\
\text { location }\end{array}$ & ZoneA & ZoneB & ZoneC & ZoneD \\
\hline $\mathrm{a}$ & 0 & $\mathrm{O} 1$ & 1584.86 & 429.43 & 1050.93 & 324.86 \\
\hline $\mathrm{b}$ & 0 & $\mathrm{O} 2$ & 2554.76 & 506.70 & 300.71 & 296.07 \\
\hline c & 0 & $\mathrm{O} 3$ & 2141.31 & 481.36 & 656.67 & 628.26 \\
\hline d & -1 & $\mathrm{O} 1$ & 1245.23 & 534.60 & 1028.82 & 358.69 \\
\hline e & -1 & $\mathrm{O} 2$ & 2462.76 & 517.71 & 434.74 & 600.93 \\
\hline $\mathrm{f}$ & -1 & $\mathrm{O} 3$ & 1853.10 & 396.94 & 300.00 & 551.69 \\
\hline g & 1 & $\mathrm{O} 1$ & 1484.93 & 350.44 & 814.88 & 608.35 \\
\hline $\mathrm{h}$ & 1 & $\mathrm{O} 2$ & 1862.90 & 458.00 & 331.00 & 455.15 \\
\hline $\mathrm{i}$ & 1 & $\mathrm{O} 3$ & 1906.74 & 481.10 & 496.53 & 876.40 \\
\hline
\end{tabular}

As shown in Table4, the difference of first fixation duration for angle level 0 and 1 was significant. This means first duration was decline when the angle was increased at zone A. The difference of first fixation duration for focus point location $\mathrm{O} 1, \mathrm{O} 2$ and $\mathrm{O} 3$ was significant. As shown in table4, the duration of $\mathrm{O} 1$ was the shortest. Under the same stripe angle, focus point location $\mathrm{O} 2$ get more people's vision at zone A.

\section{Conclusions}

Through eye tracking experiment, it was proved that the strip with increasing density can be concentrated easily. The midpoint of armhole curve attracted more people's attention than other points. This can be a reference for designing the upper limb disabled clothing with stripe.

\section{Acknowledgements}

This work was financially supported by the social sciences union of Zhejiang province (2012N195) and the science and technology planning project of Zhejiang province (2013C31142) and the Zhejiang University of Science and Technology foundation.

\section{References}

[1] M.Thoren: Applied Ergonomics Vol. 27(1996),p.389-396

[2] N. Nevala, J.Holopainen, O.Kinnunen and O. H.anninen. Applied Ergonomics Vol.34 (2003), p. 557-563

[3] R. W. Chase:Design Without Limits(Simplicity pattern Company, America 1990). 